\title{
用平均键能确定异质结价带偏移的 准确性研究*
}

\author{
王仁智 柯三黄 郑永梅 黄美纯
}

(厦门大学物理系, 厦门 361005)

\section{关键词异质结、价带偏移}

我们建议的以平均键能为参考能级的异质结价带偏移 $\Delta E_{\mathrm{v}}$ 值的理论计算方法 ${ }^{[1]}$ 已在一系 列晶格匹配的异质结的 $\Delta E_{\mathrm{v}}$ 值的理论计算中获得比一般线性理论方法 ${ }^{[2-4]}$ 更准确的结果, 几 乎达到计算量极大的界面自洽计算方法 ${ }^{5-6}$ (也称为非线性理论方法)的准确性：本文对于由 $\mathrm{AlAs}$ 和 $\mathrm{GaAs}$ 构成的 AlAs/GaAs 异质结的 (110)、(111) 以及 (100) 三种不同晶面的 (AlAs) $(\mathrm{GaAs})_{3}$ 超原胞 (简称 $\mathrm{SL}_{3}$ ), 分别采用 $E_{\mathrm{m}}$ 为参考能级的方法和界面自洽方法进行了理论计算 和比较, 全面考察 $\mathrm{SL}_{3}$ 中的平均键能 $E_{\mathrm{m}}$ 对齐程度与界面结构、界面电荷转移及计算方案的关 系, 揭示以平均键能为参考能级的 $\Delta E_{\mathrm{v}}$ 理论计算方法可达到界面自洽方法的准确性的主要原 因.

\section{AlAs 和 GaAs 晶体的能带、平均键能 $E_{\mathrm{m}}$ 的计算和 AlAs/GaAs 异质结 $\Delta E_{v}$ 值的确定}

本文采用基于局域密度泛函理论的线性 Muffin-Tin 轨道 (LMTO) 能带方法, 在 AlAs 和 $\mathrm{GaAs}$ 闪锌结构晶体的能带计算中采用 2 个特殊 $k$ 点, 取其晶格常数 $a=5.65 \AA$, 原胞体积 $\Omega=a^{3} / 4$, 原胞中包含 2 个原子球和 2 个空原子球(简称空球). 在本文计算中, 取原子球和空 球有相同的体积, 在 $\mathrm{AlAs}$ 的能带计算中, 原子球和空球取 $s, p, d$ 态, 其中 $d$ 态是未填充价电 子的 “空 $d$ 态”, 对于此空 $d$ 态, 我们引用 Löwdin 微扰法计人”) (这种计算方法简称为 L 计算 方案 ); 在 GaAs 能带计算中, 除采用上述的 L 计算方案外, 还采用另一种简称为 B 的计算方 案, 这就是考虑到 $\mathrm{Ga}$ 的 $3 d$ 电子与 $4 s$ 和 $4 p$ 能级比较接近, 将 $\mathrm{Ga}$ 的 $3 d$ 电子与 $4 s$ 和 $4 p$ 电子 一道都作为价电子态处理, 即能带计算中包含着 $d$ 能带. 采用 $\mathrm{L}$ 和 $\mathrm{B}$ 两种计算方案对 $\mathrm{AlAs}$ 和 GaAs 进行能带计算, 在所得到的能带结构 $E_{\mathrm{n}}(k)$ 中, 禁带下方的 4 个价带对应于 $s, p$ 成键 态, 禁带上方的 4 个较低导带近似地对应于 $s, p$ 反成键态. 我们采用下面的式子计算它们的 成键态能量 $E_{\mathrm{b}}$, 反成键态能量 $E_{\mathrm{a}}$ 和平均键能 $E_{\mathrm{m}}$ :

$$
E_{\mathrm{b}}=\frac{1}{l N} \sum_{n=1}^{l} \sum_{k} E_{n}(k),
$$

1994-02-14 收稿.

* 国家自然科学基金和福建省自然科学基金资助项目. 


$$
\begin{gathered}
E_{\mathrm{a}}=\frac{1}{l N} \sum_{n=l+1}^{2 l} \sum_{k} E_{n}(k), \\
E_{m}=\left(E_{\mathrm{b}}+E_{\mathrm{a}}\right) / 2,
\end{gathered}
$$

其中 $N$ 为晶体中原胞的个数, $l$ 为对应于成键态的价带数目或对应于反成键态的导带数目. 对于闪锌矿结构晶体, $l=4$. 在采用 $\mathrm{L}$ 计算方案对 $\mathrm{AlAs}$ 和 $\mathrm{GaAs}$ 以及采用 $\mathrm{B}$ 方案对 $\mathrm{GaAs}$ 进 行能带计算得到 $E_{\mathrm{n}}(k)$ 之后, 由式 (1)-(3) 计算得到的平均键能值 $E_{\mathrm{m}}$ 列于表 1. 因在 LMTO 能带方法中 $s$ 态结构常数在 $k=0$ ( $r$ 点) 处发散, 不能直接得到 $r$ 点的 $E_{\mathrm{v}}$ 值, 表 1 中的 $E_{\mathrm{v}}$ 值是 $r$ 点附近 $k=2 \pi / a(0.075,0,0)$ 处的计算结果. 根据表 1 的体材料 AlAs 和 GaAs 的 $E_{\mathrm{m}}-E_{\mathrm{v}}$ 值 的计算结果, 假设构成 $\mathrm{AlAs} / \mathrm{GaAs}$ 异质结后 $\mathrm{AlAs}$ 和 $\mathrm{GaAs}$ 的 $E_{\mathrm{m}}$ 值相同 (称之为 $E_{\mathrm{m}}$ 在异质 结中的对齐), 则由下式可计算 $\mathrm{AlAs} / \mathrm{GaAs}$ 异质结的 $\Delta E_{\mathrm{v}}$ 值.

$$
\Delta E_{\mathrm{v}}=\left[E_{\mathrm{m}}(\mathrm{AlAs})-E_{\mathrm{v}}(\mathrm{AlAs})\right]-\left[E_{\mathrm{m}}(\mathrm{GaAs})-E_{\mathrm{v}}(\mathrm{GaAs})\right],
$$

计算结果列于表 1 的 $\Delta E_{\mathrm{v}}$ 栏中, 此栏括号内的数值为界面自洽方法 $\mathrm{SCIC}^{[\Im]}$ 和 $\mathrm{SCSC}^{[6]}$ 的计算 结果, 供比较.

\begin{tabular}{|c|c|c|c|c|c|c|}
\hline 晶体结构 & 计算方来 & & $\Delta Q$ & $E_{\mathrm{m}}$ & $E_{\mathrm{v}}$ & $E_{\mathrm{m}}-E_{\mathrm{v}}$ \\
\hline $\mathrm{ZB}$ & $L$ & AlAs & 0.00 & -0.64 & -1.23 & 0.59 \\
\hline \multirow[t]{3}{*}{ ZB } & L & GaAs & 0.00 & -1.07 & -1.30 & 0.23 \\
\hline & & $\Delta E_{\mathrm{m}}$ & & 0.43 & & \\
\hline & & $\Delta E_{\mathrm{v}}$ & & & $0.36^{a \prime}\left(0.36^{b)}\right)$ & \\
\hline ZB & L & AlAs & ๑.00 & -0.64 & -1.23 & 0.59 \\
\hline \multirow[t]{3}{*}{ ZB } & B & GaAs & 0.00 & -1.05 & -1.10 & 0.05 \\
\hline & & $\Delta E_{\mathrm{m}}$ & & 0.41 & & \\
\hline & & $\Delta E_{\mathrm{v}}$ & & & $0.54^{a)}\left(0.53^{c)}\right)$ & \\
\hline
\end{tabular}

表 1 闪锌矿结构唱体 $\mathrm{AlAs}, \mathrm{GaAs}$ 的平均键能 $E_{\mathrm{m}}$, 价带顶 $E_{\mathrm{v}}$ 和 $E_{\mathrm{m}}-E_{\mathrm{v}}$ 值的计算结果 $(\Delta Q$ 值的 $\mathrm{AlAs}$ 和 $\mathrm{GaAs}$ 原胞的净带电量) $(\mathrm{eV})$

a) 由 $\mathrm{AlAs}$ 与 $\mathrm{GaAs}$ 的 $E_{\mathrm{m}}$ 对齐所确定的 $\Delta E_{\mathrm{v}}$ 值，b), c) 分别是界面自洽方法 $\mathrm{SCIC}^{19}$ 和 $\mathrm{SCSC}^{(\theta)}$ 的计算结果.

\section{2 三种不同晶面的 $(\mathrm{AlAs})_{3}(\mathrm{GaAs})_{3}$ 超原胞的界面自洽计算及其 $(\mathrm{AlAs})_{3}$, $(\mathrm{GaAs})_{3}$ 分子层的能带和平均键能 $E_{\mathrm{m}}$ 值的计算}

在 (110), (111) 和 (100) 三种不同晶面的 $(\mathrm{AlAs})_{3}(\mathrm{GaAs})_{3}$ 超原胞 (简称 $\mathrm{SL}_{3}$ ) 的能带计算 中, 我们分别采用正交、六角和四方原胞结构; 布里渊区积分分别采用 4,3 和 2 个特殊 $k$ 点. 三种 $\mathrm{SL}_{3}$ 原胞中都包含三个 $\mathrm{AlAs}$ 分子层 $(\mathrm{AlAs})_{3}$ 和 3 个 $\mathrm{GaAs}$ 分子层 $(\mathrm{GaAs})_{3}$, 每个分子层 包含有 2 个原子球和 2 个空球, 每个分子层的体积等于闪锌结构原胞的体积, 分子层中的原子 球和空球与 $\mathrm{AlAs}$ 或 GaAs 原胞中的原子球和空球存在 1- 1 对应的关系. 据此, 由 (AlAs) 3 $(\mathrm{GaAs})_{3}$ 超原胞能带自洽计算结果, 可以采用冻结势方法 ${ }^{6}$ 计算其中的 (AlAs) ${ }_{3}$ 和 (GaAs) ${ }_{3}$ 分 子层的能带结构和平均键能. 这就是, 将 $\mathrm{SL}_{3}$ 自洽计算得到的 12 个原子球和 12 个空球的ASA 势, 按超原胞结构重新组成 $(\mathrm{AlAs})_{3}(\mathrm{AlAs})_{3}$ 和 $(\mathrm{GaAs})_{3}(\mathrm{GaAs})_{3}$ 两组, 分别作为 $\mathrm{SL}_{3}$ 的 LMTO 能带计算的输人, 得到对应于 $(\mathrm{AlAs})_{3}$ 和 $(\mathrm{GaAs})_{3}$ 的能带结构, 然后通过式 (1) - (3) 计算 $(\mathrm{AlAs})_{3}$ 和 $(\mathrm{GaAs})_{3}$ 的平均键能 $E_{\mathrm{m}}$ 值. 这里需要说明的是, $\mathrm{SL}_{3}$ 原胞体积是闪锌结构原 胞体积的 6 倍, $\mathrm{SL}_{3}$ 布里渊区体积只有闪锌结构布里渊区体积的 $1 / 6$, 由于布里渊区的折叠, 
$\mathrm{SL}_{3}$ 超原胞的能带数目是闪锌结构能带数目的 6 倍. 因此在 $\mathrm{SL}_{3}$ 中, 对应于成键态的有 24 个 价带, 对应于反成键态的也有 24 个较低导带. 用式 (1)-(3) 计算 $E_{\mathrm{m}}$ 时, $l=24$. 表 2 列出了 (110), (111) 和 (100) 三种晶面的 $\mathrm{SL}_{3}$ 中的 $(\mathrm{AlAs})_{3}$ 和 $(\mathrm{GaAs})_{3}$ 的平均键能 $E_{\mathrm{m}}$, 价带顶 $E_{\mathrm{v}}$ 和 $E_{\mathrm{m}}-$ $E_{\mathrm{v}}$ 的计算结果, 其中 $\Delta E_{\mathrm{v}}$ 值是在 $\mathrm{SL}_{3}$ 能带自洽计算后直接采用冻结势方法得出的结果, 也就是 通常说的界面自洽方法的计算结果. 表 1 中的 $\Delta Q$ 值为零, 表示 $A 1 A s$ 和 $\mathrm{GaAs}$ 原胞保持电中 性, 表 2 中的 $\Delta Q$ 是构成 ( $\mathrm{AlAs})_{3}(\mathrm{GaAs})_{3}$ 超原胞时, 价电子由 ( $\left.\mathrm{AlAs}\right)_{3}$ 一侧向 $(\mathrm{GaAs})_{3}$ 一侧转 移 (称为界面电荷转移) 而使 (AlAs) ${ }_{3}$ 和 $(\mathrm{GaAs})_{3}$ 偏离电中性的结果.

表 2 (110), (111) 和 (100) 三种唱面的 (AlAs) ${ }_{3}(\mathrm{GaAs})_{3}$ 超原胞 ( $\mathrm{SL}_{3}$ ) 中的 (AlAs) ${ }_{3}$ 和 $(\mathrm{GaAs})_{3}$ 的平均键能 $E_{\mathrm{m}}$, 价带顶 $E_{\mathrm{v}}$ 以及 $E_{\mathrm{m}}$ - $E_{\mathrm{v}}$ 值的计算结果 ${ }^{\text {a) }}$

\begin{tabular}{|c|c|c|c|c|c|c|}
\hline 晶体结构 & 计算方案 & & $\Delta Q$ & $E_{\mathrm{m}}$ & $E_{\mathrm{v}}$ & $E_{\mathrm{m}}-E_{\mathrm{v}}$ \\
\hline $\mathrm{SL}_{3}(110)$ 中 & $\mathrm{L}$ & $(\mathrm{AlAs})_{3}$ & -0.077 & -0.84 & -1.41 & 0.57 \\
\hline \multirow[t]{3}{*}{$\mathrm{SL}_{3}(110)$ 中 } & L & $(\mathrm{GaAs})_{3}$ & 0.077 & -0.85 & -1.06 & 0.21 \\
\hline & & $\Delta E_{\mathrm{m}}$ & & 0.01 & & \\
\hline & & $\Delta E_{\mathrm{v}}$ & & & 0.35 & \\
\hline $\mathrm{SL}_{3}(111)$ 中 & $L$ & $(\mathrm{AlAs})_{3}$ & -0.126 & -0.88 & -1.45 & 0.57 \\
\hline \multirow[t]{3}{*}{$\mathrm{SL}_{3}(111)$ 中 } & L & $(\mathrm{GaAs})_{3}$ & 0.126 & -0.87 & -1.09 & 0.22 \\
\hline & & $\Delta E_{\mathrm{m}}$ & & -0.01 & & \\
\hline & & $\Delta E_{\mathrm{v}}$ & & & 0.36 & \\
\hline $\mathrm{SL}_{3}(100)$ 中 & L & $(\mathrm{AlAs})_{3}$ & -0.087 & -0.85 & -1.43 & 0.58 \\
\hline \multirow[t]{3}{*}{$\mathrm{SL}_{3}(100)$ 中 } & $\mathrm{L}$ & $(\mathrm{GaAs})_{3}$ & 0.087 & -0.84 & -1.07 & 0.23 \\
\hline & & $\Delta E_{\mathrm{m}}$ & & -0.01 & & \\
\hline & & $\Delta E_{\mathrm{v}}$ & & . & 0.36 & \\
\hline $\mathrm{SE}_{3}(100)$ 中 & $\mathrm{L}$ & $(\mathrm{A} \mid \mathrm{As})_{3}$ & -0.068 & -0.84 & -1.42 & 0.58 \\
\hline \multirow[t]{3}{*}{$\mathrm{SL}_{3}(100)$ 中 } & B & $(\mathrm{GaAs})_{3}$ & 0.068 & -0.85 & -0.87 & 0.02 \\
\hline & & $\Delta E_{\mathrm{m}}$ & & 0.01 & & \\
\hline & & $\Delta E_{\mathrm{v}}$ & & & 0.55 & \\
\hline
\end{tabular}

a) $\Delta Q$ 为界面电荷转移量 ( $\Delta Q$ 单位: 电子数, 能量单位: $e V)$.

\section{3 讨 论}

\section{1 (AlAs) ${ }_{3}(\mathrm{GaAs})_{3}$ 中的界面电荷转移与平均键能 $E_{\mathrm{m}}$ 的对齐}

从表 1 可看到, $\mathrm{AlAs}$ 的 $E_{\mathrm{m}}$ 值比 $\mathrm{GaAs}$ 的高, $\Delta E_{\mathrm{m}}$ 值为 $0.41-0.43 \mathrm{eV}$, 当 $\mathrm{AlAs}$ 和 $\mathrm{GaAs}$ 构 成 (AlAs) ${ }_{3}(\mathrm{GaAs})_{3}$ 超原胞时, 在 (110)、(111) 和 (110) 三种不同晶面的 $\mathrm{SL}_{3}$ 中都发生界面电荷 转移, 价电子都是从 $E_{\mathrm{m}}$ 值较高的 $(\mathrm{AlAs})_{3}$ 一侧向 $E_{\mathrm{m}}$ 值较低的 $(\mathrm{GaAs}$ )一侧转移 (见表 2 中 $\Delta Q$ 值), 界面电荷转移使 (AlAs) ${ }_{3}$ 的 $E_{\mathrm{m}}$ 值降低; $(\mathrm{GaAs})_{3}$ 的 $E_{\mathrm{m}}$ 值提高, 最后使超原胞中的 $(\mathrm{AlAs})_{3}$ 和 ( $\left.\mathrm{GaAs}\right)_{3}$ 的 $E_{\mathrm{m}}$ 值趋于一致 (称之为 $E_{\mathrm{m}}$ 对齐).

\section{-3.2 AlAs/GaAs 异质结构键能对齐的普适行为}

在表 2 的三种不同晶面的 (AlAs) ${ }_{3}(\mathrm{GaAs})_{3}$ 中, (100) 和 (111) 是极性面, (110) 为非极性面, 三种异质界的结构不同, 因而界面电荷转移量 $\Delta Q$ 值也有所差别, 但是在三种不同的 $\mathrm{SL}_{3}$ 中, 在 L. 计算方案下 $E_{\mathrm{m}}$ 的对齐程度都非常好 $\left(\Delta E_{\mathrm{m}}\right.$ 值均为 $\left.0.01 \mathrm{eV}\right)$; 还可以看到, 对于 (100) 晶面 情况, 将 $\mathrm{Ga}$ 原子球的 $d$ 态处理方法由 $\mathrm{L}$ 计算方案改为 $\mathrm{B}$ 计算方案时, 虽然 $E_{\mathrm{m}}$ 值有所改变, 住 
$E_{\mathrm{m}}$ 的对齐程度不受影响 (仍旧保持 $E_{\mathrm{m}}$ 为 $0.01 \mathrm{eV}$ ). 也就是说, $\mathrm{AlAs} / \mathrm{GaAs}$ 异质结中的 $E_{\mathrm{m}}$ 的 对齐程度基本上不受界面结构的影响, 也不会因为对 $\mathrm{Ga}$ 原子的 $d$ 态处理方法的不同而改 变, 因此, 我们认为, 平均键能对齐是异质结中的一个普适行为.

\section{3 界面结构和界面电荷转移对 $E_{\mathrm{m}}-E_{\mathrm{v}}$ 值的影响较小}

表 1 中 $\mathrm{AlAs}$ 和 $\mathrm{GaAs}$ 的 $E_{\mathrm{m}}-E_{\mathrm{v}}$ 值是原胞保持电中性 $(\Delta Q=0)$ 情况下的计算结果, 表 2 (AlAs) ${ }_{3}$ 和 (GaAs) ${ }_{3}$ 的 $E_{\mathrm{m}}-E_{\mathrm{v}}$ 值是在 $\mathrm{SL}_{3}$ 中发生界面电荷转移情况下 $(\Delta Q \neq 0)$ 的计算结果. 可 以看到, 对于 $\mathrm{AlAs}$ 的 $E_{\mathrm{m}}-E_{\mathrm{v}}$ 值, 表 1 给出的数值为 $0.59 \mathrm{eV}$, 对于 (AlAs) ${ }_{3}$ 的 $E_{\mathrm{m}}-E_{\mathrm{v}}$ 值, 表 2 的 数值为 $0.57-0.58 \mathrm{eV}$ 体材料和超原胞中的 $E_{\mathrm{m}}-E_{\mathrm{v}}$ 值差别很小 (为 $0.01 \mathrm{eV}-0.02 \mathrm{eV}$ ); 对于 $\mathrm{GaAs}$ 和 $(\mathrm{GaAs})_{3}$ 在 $\mathrm{L}$ 和 $\mathrm{B}$ 两种计算方案下得到的 $E_{\mathrm{m}}-E_{\mathrm{v}}$ 值, 表 1 为 $0.23 \mathrm{eV}$ 和 $0.05 \mathrm{eV}$, 表 2 为 $0.21 \mathrm{eV}-0.23 \mathrm{eV}$ 和 $0.02 \mathrm{eV}$, 表 1 和表 2 的数值相差也很小 (约为 $0.01-0.03 \mathrm{eV}$ ). 可 见, 异质结界面结构和界面电荷转移对构成异质结前后的 AsAs 和 GaAs 的 $E_{\mathrm{m}}-E_{\mathrm{v}}$ 值的影响甚 小. 这是因为界面电荷转移主要引起异质结两侧的能带的整体相对平移, 基本上不改变它们 的能带结构的特征.

\section{4 以 $E_{\mathrm{m}}$ 为参考能级的 $\Delta E_{\mathrm{v}}$ 理论计算方法的准确性}

根据单独体材料 $\mathrm{AlAs}$ 和 $\mathrm{GaAs}$ 的 $E_{\mathrm{m}}-E_{\mathrm{v}}$ 值的计算结果, 由式 (4) 计算 $\mathrm{AlAs} / \mathrm{GaAs}$ 异质结 的 $\Delta E_{\mathrm{v}}$ 值, 其准确性取决于两方面：1) $\mathrm{AlAs} / \mathrm{GaAs}$ 异质结中 $E_{\mathrm{m}}$ 值的真实对齐程度；2) 式 (4) 计算的 $\Delta E_{\mathrm{v}}$ 值是 $\mathrm{AlAs} / \mathrm{GaAs}$ 异质结中的价偏移, 因界面结构和界面电荷转移的作用, $\mathrm{AlAs}$ 和 $\mathrm{GaAs}$ 构成 $\mathrm{AlAs} / \mathrm{GaAs}$ 异质结前后, 它们的 $E_{\mathrm{m}}-E_{\mathrm{v}}$ 值可能发生改变, 其变化量将影响 $\Delta E_{\mathrm{v}}$ 值的准确性. 上面的研究结果已着重阐明: 在 $\mathrm{AlAs} / \mathrm{GaAs}$ 异质结中, $E_{\mathrm{m}}$ 对齐程度极好且 不受晶面取向计算方法改变的影响; 并且体材料 $\mathrm{AlAs}$ 和 $\mathrm{GaAs}$ 的 $E_{\mathrm{m}}-E_{\mathrm{v}}$ 值与 $\mathrm{SL}_{3}$ 中的 $(\mathrm{AlAs})_{3}$ 和 $(\mathrm{GaAs})_{3}$ 的 $E_{\mathrm{m}}-E_{\mathrm{v}}$ 值相当接近, 所以, 以平均键能 $E_{\mathrm{m}}$ 为参考能级的 $\Delta E_{\mathrm{v}}$ 理论计算方 法可能得出与界面自洽计算方法一致的计算结果．例如, 表 1 的 L、L 计算方案和 L、B 计算方 案的 $\Delta E_{\mathrm{v}}$ 值为 $0.356 \mathrm{eV}$ 和 $\left.0.54 \mathrm{eV}\right)$ 与本文相应计算方案下的界面自洽计算结果 $(0.36 \mathrm{eV}$ 和 $0.55 \mathrm{eV}$ ) 相当一致,也和文献[5] 采用从头赝势方法和文献[6] 采用LMTO 方法的界面自洽计算 结果相当吻合.

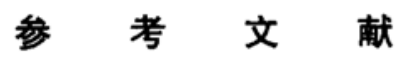

(1) 王仁智.黄美纯, 中国科学, A 辑, 1992, (10): 1073.

[2] Harrison, W. A., Tersoff, J., J. Vac. Sci. Technol., 1986, B4: 1068.

[3] Tersoff, J., J. Vac. Sci. Technol., 1986, B4: 1066.

[4] Cardona, M., Christensen, N. E., Phys. Rev., 1987, B35: 6182.

[5] Van de Walle, C. G., Martin, R. M., Phys. Rev., 1987, B35: 8154 .

[6] Christensen, N. E., Phys. Rev., 1988, B37: 4528.

[7] 王仁智、黄美纯、计算物理, 1990, 7:85. 